\title{
SEMI-OPEN RHINOPLASTY FOR APPROACHING NASAL TIP AND DORSUM
}

\author{
Nabil Galal Zeid M.D., Mahmoud Sayed Elfouly M.D., Ahmed Ahmed Kamel M.D., Basim Metwally \\ Wahba M.D., Refaat Nashed Bahman M.D., Mohamed Abd Elmottaleb Sabaa M.Sc. \\ Otorhinolaryngology Department - Faculty of Medicine - Cairo University - Egypt.
}

Corresponding
Author:
Mohamed Abd
Elmottaleb Sabaa
01114114699
Dr.mottaleb@gma
il.com

Corresponding

Author:

Mohamed Abd

01114114699

il.com 
direct view of the nasal structure, resulting in improved precision in modeling the cartilages. However, the absence of intact skin cover exposes the surgeon to a less precise overall aesthetic evaluation. For this reason, it is not the skin scar on the columella, which usually is hardly visible, that deters the use of open rhinoplasty, but rather the reduced ability for intraoperative evaluation of the nasal proportions ${ }^{[3]}$.

The "semi-open" technique allows very wide intra-operative visual field, equivalent to that which can be achieved using the open technique, but without leaving any external scar. It has the several advantages of the open technique, and it does not involve the presence of postsurgical scars ${ }^{[4]}$.

\section{SUBJECTS AND METHODS}

This study was a prospective study, conducted in Kasralainy - Cairo University hospital over a period of 18 months starting from July 2014 to December 2015. Fifteen patients with nasal deformity candidate for rhinoplasty were recruited for this study.

\section{Inclusion criteria were as follows:}

- Crooked nose

- Nasal hump

- Nasal tip deformities

Exclusion criteria were as follows:

- Recent nasal trauma (within last 3 months).

- Medical contraindication to surgery.

\section{Surgical access}

The surgery was carried out under general anaesthesia. 1:200,000 adrenaline was injected into the nasal bridge and the alar cartilages. The vibrissae were shaved.

Semi-open technique was done through marginal incision, the LLC was dissected in a direction from lateral to medial (Fig.1A). The same steps were done on the opposite side (Fig.1B), and both medial crura were dissected from the overlying columella (Fig 1C). Domes were dissected and presented through one nostril (Fig.1D). Retraction of the dissected domes allowed dissection to be continued over the dorsal surface of the ULC and bony nasal dorsum (Fig.2A). With dissection of the skin cover from the nasal skeleton, any type of rhinoplasty can be done to the tip (Fig.2B) or nasal dorsum. 

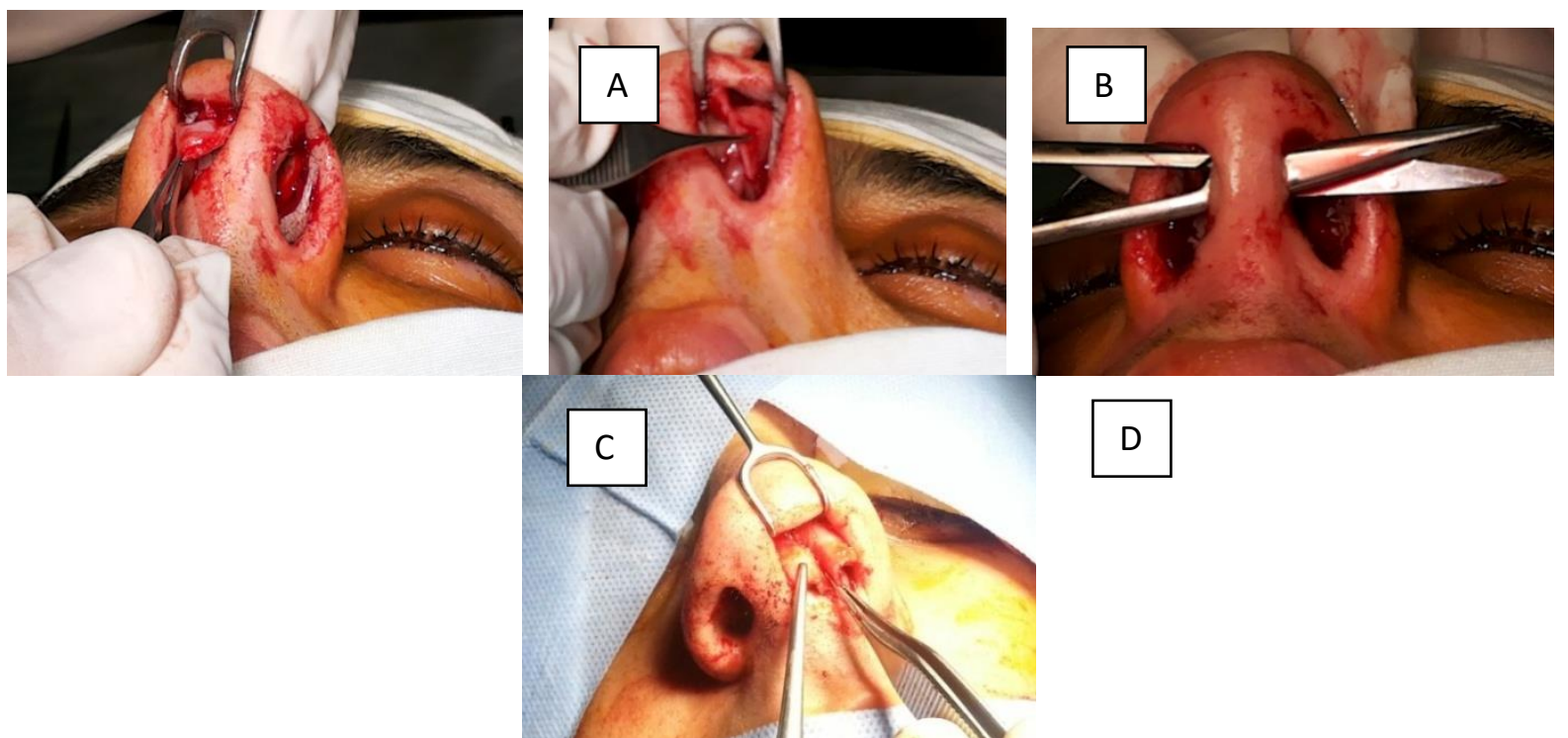

Fig. 1: Steps of performing semi-open approach in patients of group B. (A) Marginal incision was done and dissection of right LLC began. (B) Dissection of left LLC. (C) Dissection of both medial crura from the overlying skin of columella. (D) Complete dissection of both LLC as they were appearing from the left nostril.

* LLC: Lower Lateral Cartilage.

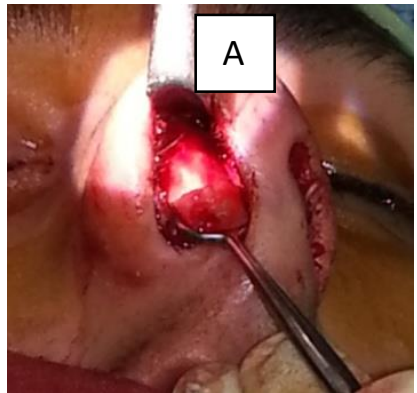

Fig. 2: (A)View of the nasal dorsum after Columellar strat fixed between both medial crura.

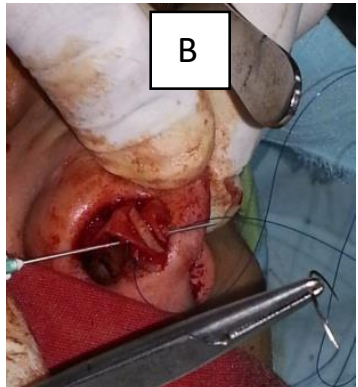

dissection and retraction of the LLC. (B)

\section{Postoperative care:}

All patients had external nasal splints for 1 week, merocel packs were removed on the 2nd day, Amoxicilin Clavulante tablets 1 $\mathrm{gm} / 12 \mathrm{hrs}$ and Paracetamol 500mg every $8 \mathrm{hrs}$ were received for 1 week.

\section{Assessment parameters:}

Patients were evaluated intraoperatively for:

- Operative time in minutes from first incision to last closing stitch.
- Accessibility for the entire lower lateral cartilages / tip complex and exposure of the dorsum. Accessibility was scored according to: (1) poor, (2) fair and (3) good.

- Oedema at the end of the operation was scored according to: (0) none, (1) mild, (2) moderate and (3) marked.

- Operative score is calculated as the sum of different steps required in the operation as follows: 
- Hump removal (1).

- Osteotomies (1).

- Tip modification (1).

- Septoplasty (1).

- Turbinate reduction (1).

Postoperative evaluation was done on 7 th day and 3 months postoperatively for:

- Residual oedema which is scored as described before.

- Scars.

- Patient's Visual Analogue Scale (VAS) which were graded as (1) very poor, (2) poor, (3) fair, (4) good and (5) excellent.

- Surgeon's VAS which were graded as patient's VAS.
Data were coded and entered using the statistical package SPSS (Statistical Package for the Social Science; SPSS Inc., Chicago, IL, USA)version 22. Data were summarized using mean, standard deviation, median minimum and maximum in quantitative data and using frequency (count) and relative frequency (percentage) for categorical data ${ }^{[5]}$.

\section{RESULTS}

\section{Demographic data:}

This study involved 15 patients with nasal deformity. Ten cases were males and 5 cases were females (Fig.3), with ages ranging from 18 to 57 years with a mean age of $26.3 \pm 10.1 \mathrm{SD}$.

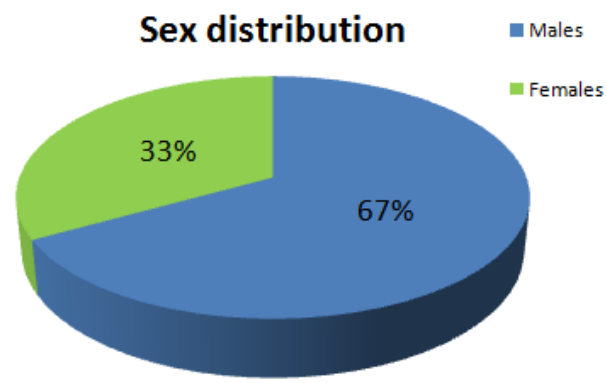

Fig. 3: Showing sex distribution among the study's population.

Preoperative nasal deformities were: Eight cases with hump (53\%), 7 cases with nasal twist (47\%), 9 cases with septal deviation (60\%), 12 cases with tip deformities (80\%), 4 cases with broad nose $(27 \%)$ and 1 case with saddle nose $(7 \%)$.

Eight operations (out of 15) were performed by the same surgeon. All patients had no previous rhinoplasty operations.

Semi-open approach showed good accessibility to dorsum in all cases. While in tip accessbility, good accessibility to the tip was achieved in all cases except one case in which tip accessibility was fair (Table 1). 
Table 1: Dorsum and tip accessibility assessment.

\begin{tabular}{|c|c|c|c|}
\hline & & \multicolumn{2}{|c|}{ Semi-Open approach } \\
\hline & & Count & $\%$ \\
\hline \multirow{3}{*}{$\begin{array}{c}\text { Tip } \\
\text { Accessibility }\end{array}$} & poor & 0 & $0 \%$ \\
\hline & fair & 1 & $6.7 \%$ \\
\hline & Good & 14 & $93.3 \%$ \\
\hline $\begin{array}{c}\text { Dorsum } \\
\text { Accessibility }\end{array}$ & Good & 15 & $100 \%$ \\
\hline
\end{tabular}
average time was $109 \pm 44 \min$ (Table 2).

Table 2: Assessment of operative time.

\section{Semi-open approach}

\begin{tabular}{cccccc} 
& Mean & SD & Median & Minimum & Maximum \\
\hline Time/min & 109.47 & 44.01 & 96.00 & 54.00 & 210.00
\end{tabular}

Eight cases (53\%) had moderate immediate and 1 week postoperative edema, while 7 cases (47\%) still had residual mild edema 3 months postoperatively (Table 3 ).

Table 3: Edema assessment: immediately postoperative, 1week and 3 months follow up periods.

\begin{tabular}{ccccccc}
\hline \multirow{2}{*}{ Edema } & \multicolumn{2}{c}{ Immediate } & \multicolumn{2}{c}{ 1 week } & \multicolumn{2}{c}{ 3 months } \\
\cline { 2 - 7 } & Count & $\%$ & Count & $\%$ & Count & $\%$ \\
\hline none & 0 & $0 \%$ & 0 & $0 \%$ & 3 & $20.0 \%$ \\
\hline mild & 3 & $20.0 \%$ & 4 & $26.7 \%$ & 7 & $46.7 \%$ \\
\hline moderate & 8 & $53.3 \%$ & 8 & $53.3 \%$ & 4 & $26.7 \%$ \\
\hline marked & 4 & $26.7 \%$ & 3 & $20.0 \%$ & 1 & $6.7 \%$ \\
\hline
\end{tabular}

Patient's and doctor's VAS average were approximately 4 denoting good grade (Table 4). 
Table 4: Patients' and doctor's VAS in the period of 1 week and 3 months postoperative follow up period.

\begin{tabular}{lccccc}
\multicolumn{7}{c}{} & \multicolumn{5}{c}{ Semi-Open approach } \\
\cline { 1 - 4 } & Mean & SD & Median & Minimum & Maximum \\
\hline $\begin{array}{l}\text { 1w P } \\
\text { VAS }\end{array}$ & 3.67 & 0.82 & 3.00 & 3.00 & 5.00 \\
\hline $\begin{array}{l}\text { 1w D } \\
\text { VAS }\end{array}$ & 4.27 & 0.46 & 4.00 & 4.00 & 5.00 \\
\hline $\begin{array}{l}\text { 3m P } \\
\text { VAS }\end{array}$ & 3.87 & 0.92 & 4.00 & 2.00 & 5.00 \\
\hline $\begin{array}{l}\text { 3m D } \\
\text { VAS }\end{array}$ & 4.27 & 0.70 & 4.00 & 3.00 & 5.00 \\
\hline
\end{tabular}

* 1w P VAS: 1 week patients' visual analogue scale, 1 w D VAS: 1 week doctor's visual analogue scale, 3m P VAS: 3 months patients' visual analogue scale and 3m D VAS: 3 months doctors visual analogue scale.

Four patients had nasal scars within the study's population, which were preoperative previous scars not related to the surgery done except one patient who developed post operative alar scar as a result of partial wedge resection of the alae.

Six operations done were in the operative score $3(40 \%)$ so further statistical analysis was done for this group of operation score to minimize operation's variability.

Both tip and dorsum accessibility were good in all cases within operative score 3 group.

Semi-open approach average operative time in "operative score 3 group" were 117 \pm 63 min (Table 5).

Table 5: Operative time assessment in "operative score 3 group".

\begin{tabular}{lccccc}
\hline & & & & \\
& & & \\
& & & & \\
& Mean & SD & Median & Minimum & Maximum \\
\cline { 2 - 5 } Time/min & 116.67 & 62.53 & 89.00 & 57.00 & 210.00 \\
\hline
\end{tabular}

Three cases $(50 \%)$ had marked immediate postoperative edema, 4 cases $(67 \%)$ had moderate postoperative edema 1 week post operatively and 4 cases (67\%) still had residual mild postoperative edema after 3 months interval (Table 6). 
Table 6: "Operative score 3 group" edema assessment: immediately postoperative, 1week and 3 months follow up periods.

\begin{tabular}{ccccccc}
\hline \multirow{2}{*}{ Edema } & \multicolumn{2}{c}{ Immediate } & \multicolumn{2}{c}{ 1 week } & \multicolumn{2}{c}{3 months } \\
\cline { 2 - 7 } & Count & $\%$ & Count & $\%$ & Count & $\%$ \\
\hline None & 0 & $0 \%$ & 0 & $0 \%$ & 1 & $16.7 \%$ \\
\hline Mild & 1 & $16.7 \%$ & 1 & $16.7 \%$ & 4 & $66.7 \%$ \\
\hline Moderate & 2 & $33.3 \%$ & 4 & $66.7 \%$ & 0 & $0 \%$ \\
\hline Marked & 3 & $50.0 \%$ & 1 & $16.7 \%$ & 1 & $16.7 \%$ \\
\hline
\end{tabular}

Patient's and doctor's VAS average were approximately 4 denoting good grade (Table 7).

Table 7: "Operative score 3 group" assessment of patients's VAS and doctor's VAS in the period of 1 week and 3 months postoperative follow up period.

\section{Semi-Open approach}

\begin{tabular}{lccccc}
\hline & Mean & SD & Median & Minimum & Maximum \\
\hline 1w P & 3.50 & 0.84 & 3.00 & 3.00 & 5.00 \\
VAS & 4.17 & 0.41 & 4.00 & 4.00 & 5.00 \\
\hline $\begin{array}{l}\text { 1w D } \\
\text { VAS }\end{array}$ & 4.83 & 0.75 & 4.00 & 3.00 & 5.00 \\
\hline $\begin{array}{l}\text { 3m P } \\
\text { VAS }\end{array}$ & & & 4.00 & 5.00 \\
\hline $\begin{array}{l}\text { 3m D } \\
\text { VAS }\end{array}$ & 4.50 & 0.55 & 40 & 4.00 & \\
\hline
\end{tabular}

* 1w P VAS: 1 week patients' visual analogue scale, 1 w D VAS: 1 week doctor's visual analogue scale, 3m P VAS: 3 months patients' visual analogue scale and 3m D VAS: 3 months doctors visual analogue scale. 

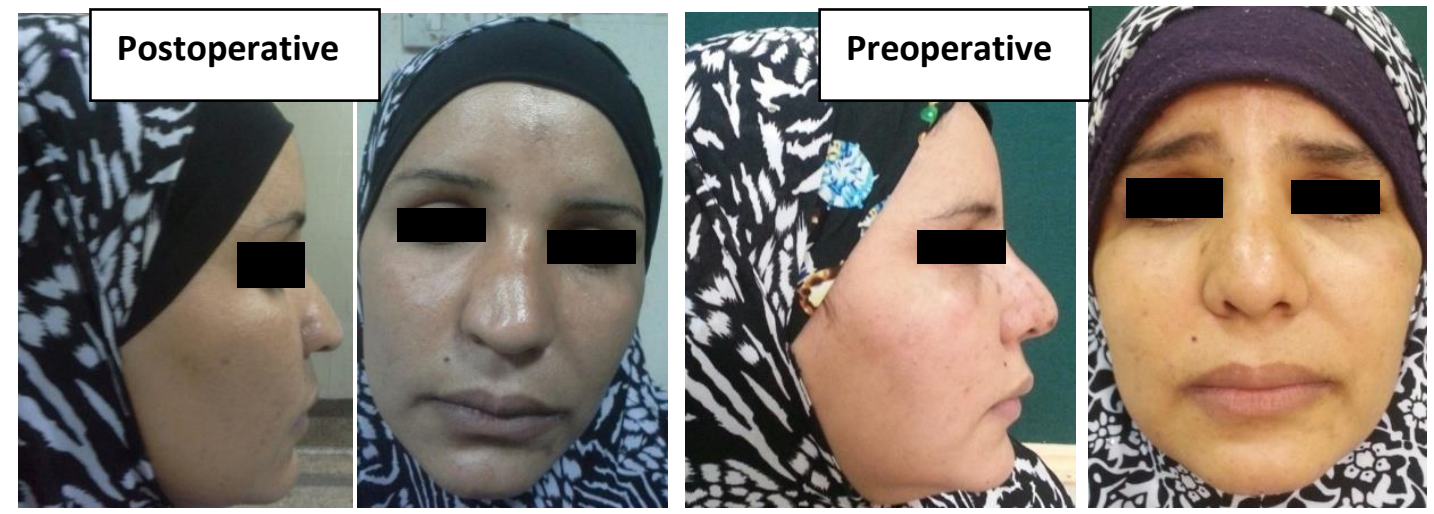

Fig. 4: Preoperative and post operative pictures. Hump rasping, median, lateral osteotomies, intradomal suturing, interdomal suturing, dorsal cartilage augmentation were done. Fair tip accessibility was achieved in this case due to small sized nostrils.
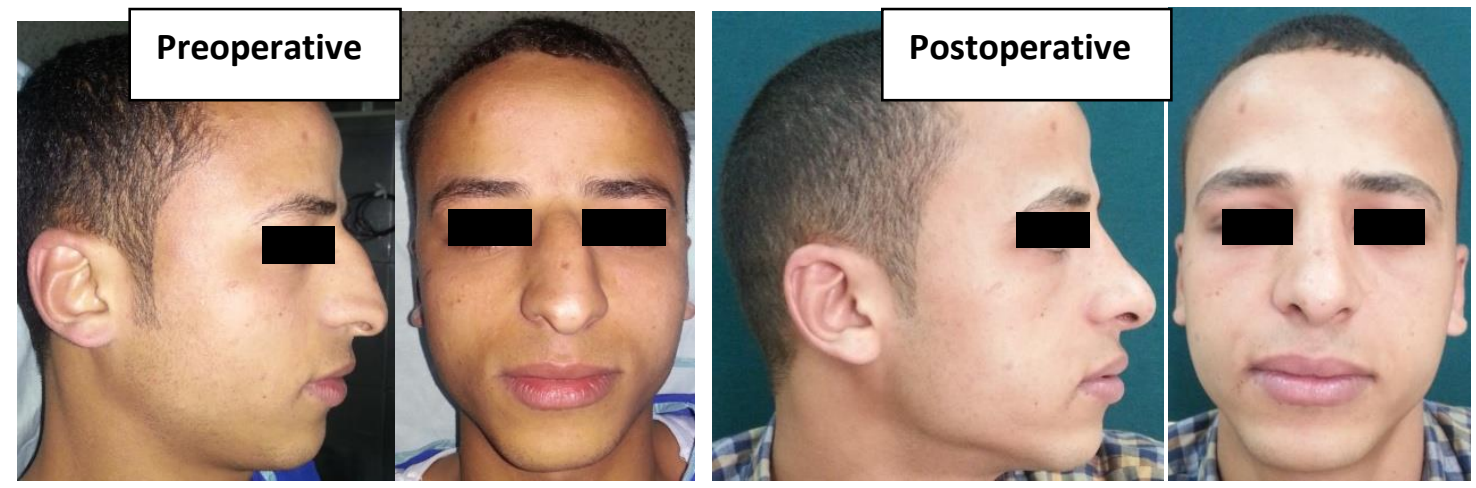

Fig. 5: Preoperative and post operative pictures. Removal of nasal hump and lateral osteotomies were done.

\section{DISCUSSION}

The "open" (external) and "closed" (endonasal) rhinoplasty approaches remain a subject for debate ${ }^{[2]}$.

External approach rhinoplasty allows the surgeon to visualize, assess, and modify the structures of the nose under an "open sky". Advantages of this "external" approach include full visualization of the operative field that allows the surgeon and assistant/ trainee to appreciate the surgical steps with the ability to create minute changes to the tip. However, there is a rarely visible columellar incision line ${ }^{[6]}$.

On the contrary, in endonasal approaches there are no external incisions, and there is less dissection required; therefore, soft tissue trauma and scarring are minimized [2].

Semi-open approach was described by Inchingolo et al. ${ }^{[4]}$ in 2012 with the advantages of the open technique, and it does not involve the presence of postsurgical scars.

Accessibility to dorsum was good in all cases while tip accessibility was good in 14 cases representing $93 \%$ of cases and fair in 1 case (7\% of cases), which could be explained by the small sized nostril the patient had (Fig.4), resulting in a less tip accessibility. Kim and Park ${ }^{[7]}$ in 2013 described very small nostrils as a contraindication to semi-open approach.

Critics of open rhinoplasty have reported prolonged tip edema and extended 
operating time ${ }^{[8]}$, which matched this study results. Time factor was relatively longer with a mean of 109 minutes if compared to closed approaches in another study conducted on 15 patients in Kasralainy in the same time interval where the mean operative time was 59 minutes.

Most of cases $(53 \%)$ had moderate edema immediately and 1 week postoperatively which was prolonged as $47 \%$ of cases had residual mild edema after 3 months follow up.

In contrast to this study, Kim and Park in 2013 in their study reported less postoperative swelling as an advantage of the semi-open approach. However, no evidence and no explanation were given.

There were no postoperative scars except in one case, where partial resection of the alae was performed.

Mean patient's visual analogue scale (VAS out of 5), at 1 week and 3 months postoperative intervals were 3.67 and 3.87 respectively, while the mean doctor's VAS at 1 week and 3 months intervals were 4.27 in both of them. Therefore, both 1 week and 3 months interval nearly had similar results with a VAS of approximately 4 (good). Kamburoglu and Kayıkcoglum [9] in 2014 in their study also reported high satisfaction rate $(96 \%)$ for the semi-open approach.

Rhinoplasty remains one of the most difficult procedures to perform in plastic surgery. There are multiple anatomic components and factors that need to be managed during surgery ${ }^{[10]}$. Each rhinoplasty is different from one patient to another due to many variables around and within each operation as for example, the surrounding environment of the operation theatre, instruments, surgeon, anesthesiologist, assistant, patient's pulse, blood pressure, intra-operative bleeding state, deformity and anatomical variations. Operation score was proposed in this study to minimize variations regarding intraoperative management. Since most of the patients were in the operation score 3 representing $40 \%$ of cases, further statistical analysis was done for the population within this operation score.

Considering the study population with operative score 3, tip and dorsum accessibility was good. Operative time was relatively longer with a mean of $117 \mathrm{~min}$ if compared to closed approaches in another study conducted in Kasralainy hospital in the same time interval where the mean operative time of score 3 patients was 65 min. Immediate postoperative edema levels were marked in $50 \%$ of cases, $67 \%$ of cases had moderate edema at 1 week follow up period. Edema levels were prolonged in most of cases and $67 \%$ of cases continued to have residual edema at 3 months interval.

Bruschi et al. ${ }^{[3]}$ in 2006 added another advantage to the approaches using marginal incision, which is the more precise intra-operative assessment of the nasal shape as there is overlying skin cover and no need for frequent columellar closure for intra-operative assessment.

Kim and park [7] in 2013 noted a major disadvantage to transcolumellar incision as the columellar artery is sacrificed in the transcolumellar incision, the lateral nasal arteries become the main blood supply for the nasal tip. In a bulbous fatty tip, defatting is a mandatory procedure in the nasal tip, and the lateral nasal artery can be easily damaged. If both the lateral nasal arteries are damaged in an open approach with transcolumellar incision, the nasal tip can be compromised and become necrotic. Even a hematoma on the nasal tip, which can compress both lateral nasal arteries, may contribute to an unstable blood supply in the nasal tip which can ultimately result in necrosis.

\section{CONCLUSION}

Semi-open approach allows good exposure to tip and dorsum without dividing the columella which can be used in cases with nasal dorsum and tip deformities. 
However, it is time consuming with prolonged postoperative edema in many cases.

Further comparative studies with larger sample size and for longer follow up period are recommended to assess its long term effect. Future studies can include objective nasal flow tests to assess its effect on the nasal air flow.

\section{REFERENCES}

1- Cingi $\mathbf{C}$ and Eskiizmir G. Deviated nose attenuates the degree of patient satisfaction and quality of life in rhinoplasty: a prospective controlled study. Clin. Otolaryngol 2013; 38:136-141.

2- Burke A and Cook T. Open versus closed rhinoplasty: what have we learned? Current Opinion in Otolaryngology \& Head and Neck Surgery 2000;8:332-336.

3- Bruschi S, Bocchiotti M, Verga M, Kefalas N, Fraccalvieri M. Closed Rhinoplasty with Marginal Incision: Our Experience and Results. Aesth. Plast. Surg. 2006;30:155158.

4- Inchingolo F, Tatullo $M$, Marrelli $M$, Inchingolo D, Corelli R, et al. Semi-open rhinoplasty: a new Maxillofacial technique. Head \& Face Medicine 2012;8:(13):1-6.

5- Chan Y. Biostatistics 103: Qualitative Data -Tests of Independence. Singapore Med J. 2003b;44(10): 498-503.

6- D'Ascanio L. “Closed" Rhinoplasty: an Outof-Date Procedure? Ann Otolaryngol Rhinol 2014;1(2): 1008-1013.

7- Kim P and Park D. Advanced Aesthetic Rhinoplasty Art, Science, and New Clinical Techniques. Chapter 21: External Marginal Approach Rhinoplasty (EMA Rhinoplasty) 2013: 241- 295.

8- Gunter J, Rohrich R, Lee M, Ahmad J. Dallas Rhinoplasty.Chapter 17 Basic Nasal Tip Surgery: Anatomy and Technique 2014:347.

9- Kamburoglu $H$ and Kayıcioglum A. Closed Rhinoplasty with Open Approach Advantages: Extended Intranasal Incisions and Tip Rearrangement Sutures. Aesth Plast Surg 2014;38(4):653-661.

10- Dayan S. Evolving techniques in rhinoplasty. Facial Plast Surg 2007;23(1):62-69.lj 